\title{
MINKOWSKIAN DISTRIBUTION OF DISCS
}

\author{
L. FEJES TÓTH
}

We recall Minkowski's famous theorem: If an open convex domain, symmetric about a lattice-point of a unit lattice contains no lattice point other than its center then its area is $\leqq 4$.

We must pay tribute to Minkowski for recognizing the significance of such a simple theorem in number theory. For the theorem becomes almost trivial by reformulating it as follows: If a lattice of open centro-symmetric convex domains has the property that none of the domains contains the center of another one then the density of the lattice is $\leqq 4$. To see this we reduce each domain about its center by a similarity in the ratio $1: 2$, obtaining a new lattice of disjoint domains. The density of this lattice is, on the one hand, $\leqq 1$, on the other hand, one quarter of the density of the original lattice.

In what follows we will call a centro-symmetric convex domain a disc. We shall say that a set of discs form a Minkowskian distribution (or arrangement) if none of the discs contains in its interior the center of another one. First we will make some remarks concerning Minkowskian distributions of general discs. Then we will prove that the densest Minkowskian circle-arrangement consists of equal circles.

Minkowski's theorem immediately implies the following

REMARK 1. If in a Minkowskian distribution of discs the centers constitute a lattice, then the density of the distribution is $\leqq 4$.

The above proof of Minkowski's theorem yields

REMARK 2. The density of a Minkowskian arrangement of homothetic (similar and similarly situated) discs is $\leqq 4$.

REMARK 3. There is no uniform upper bound for the density of Minkowskian distributions of discs.

The following proof, which is a modification of my original one, is due to M. N. Bleicher.

Let $k$ be an arbitrary positive integer. We claim that a square can be covered $k$-times by rectangles lying in the square and forming a Minkowskian arrangement. The proof goes by induction. For $k=1$ the statement being obvious, we suppose its validity for $k$. To distinguish the rectangles occurring in the inductive supposition from those added in the next step, we will call them oblongs and strips, respectively. We decompose the square $Q$ into four partial squares and construct to each of them a $k$-fold Minkowskian covering by ob-

Received by the editors June 3, 1964. 
longs lying in the respective partial square. Then we can decompose $Q$ into parallel strips in such a way that none of them contains in its interior the center of an oblong. Since, on the other hand, the center of each strip is a common boundary point of two partial squares, it cannot be contained in the interior of an oblong. Thus the oblongs and the strips together constitute a $(k+1)$-fold Minkowskian covering of $Q$.

Is there a uniform upper bound for the densities of all Minkowskian distributions of congruent discs? This problem is not solved as yet. The following remarks show that, if there is a Minkowskian distribution of congruent discs having a great density (say $10^{3}$ ), then the discs must be very thin, they must occur in many different orientations and the centers must be arranged very irregularly, in a certain sense.

Remark 4. If in a Minkowskian distribution of congruent discs, $A$ is the area of a disc and $a$ is the area of its incircle then the density of the distribution is $\leqq 4 A / a$.

This is obvious by applying Remark 2 to the incircles of the discs which also form a Minkowskian distribution.

REMARK 5. If in a Minkowskian distribution of congruent discs the discs have at most $n$ different orientations then the density of the distribution is $\leqq 4 n$.

REMARK 6. If in a Minkowskian distribution of congruent discs the set of centers is the union of $n$ lattices then the distribution has a density $\leqq 4 n$.

Remarks 5 and 6 are immediate consequences of Remark 2 and 1, respectively.

Now we turn our attention to Minkowskian circle-arrangements. According to Remark 2 the density of such an arrangement is $\leqq 4$. We will show that this bound can be replaced by $2 \pi / \sqrt{ } 3=3.627 \cdots$. This is the density of a set of congruent circles each containing besides its own center exactly six other centers equally spaced on its boundary (Figure 1). We restrict ourselves to circles whose radii $r_{1}, r_{2}, \cdots$ have a positive lower and a finite upper bound: $r=\inf r_{i}>0, R=\sup r_{i}$ $<\infty$. Introducing the homogeneity $r / R$ of the circles, this condition can be expressed by saying that the homogeneity of the circles is positive. As the main result of this paper we now can formulate the following

THEOREM 1. The density of a Minkowskian circle-distribution with positive homogeneity is always $\leqq 2 \pi / \sqrt{ } 3$.

Let the circles $c_{1}, c_{2}, \cdots$ with centers $O_{1}, O_{2}, \cdots$ and radii 


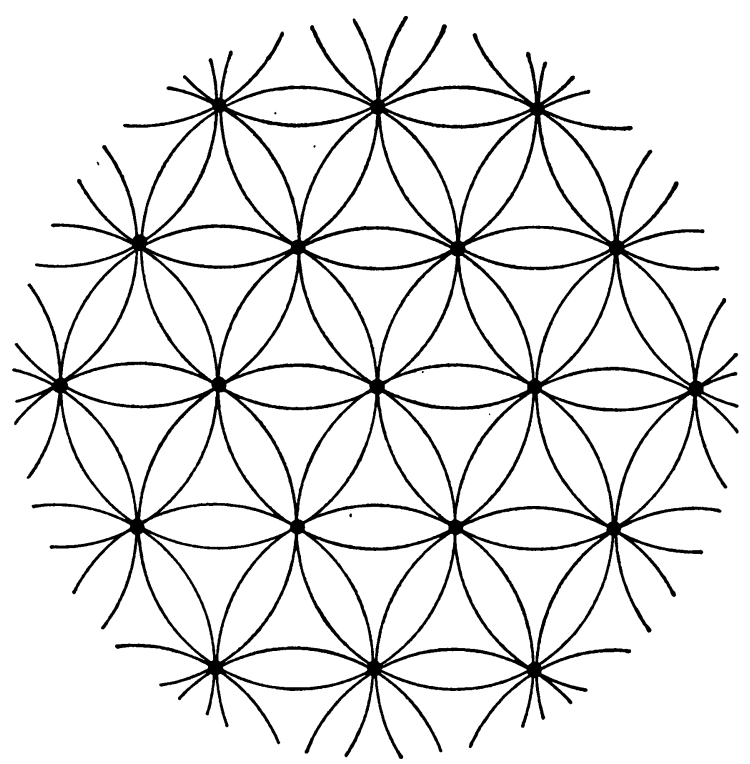

FIGURE 1

$r_{1}, r_{2}, \ldots$ form a Minkowskian arrangement with positive homogeneity. We may suppose that the plane is completely covered by the interiors of the circles. Otherwise we could successively add to the circles new ones of radius $r=\inf r_{i}$ so as to obtain a new Minkowskian circle-arrangement with density no smaller and the same homogeneity as the original one and having the desired property.

Consider the set $S_{i}$ of all points $P$ whose (algebraic) distance from $c_{i}$, defined by $d\left(P, c_{i}\right)=P O_{i}-r_{i}$, is less than or equal to their distance from any other circle $c_{k} \neq c_{i}$ :

$$
d\left(P, c_{i}\right) \leqq d\left(P, c_{k}\right), \quad k \neq i .
$$

$\left(S_{i}\right.$, being bounded by arcs of hyperbola focused at $O_{i}$, is a star region with respect to $O_{i}$.)

Obviously, the sets $S_{1}, S_{2}, \cdots$ form a tessellation $T$. Let $V$ be a vertex of this tessellation and $S_{1}, \cdots, S_{k}$ the faces around $V$. We claim that $O_{1}, \cdots, O_{k}$ are the vertices of a convex polygon $P$ which, besides its vertices, does not contain any other center $O_{i}$. To see this we observe that, in view of $d\left(V, c_{1}\right)=\cdots=d\left(V, c_{k}\right)=-\rho<0$, the circle with radius $\rho$ and center $V$ touches the circles $c_{1}, \cdots, c_{k}$ from inside. Therefore, if $O_{j} O_{l}$ is any side of the convex hull $H$ of the points $O_{1}, \cdots, O_{k}$, the triangle $V O_{j} O_{l}$ is completely covered by the 
interiors of the circles centered at its vertices. Thus $H$ cannot contain any center different from its vertices, showing that $P \equiv H$.

This property of $T$ enables us to construct the dual tessellation $T^{\prime}$, whose faces are the polygons $P$ belonging to the vertices of $T$. If a face of $T^{\prime}$ has more than three sides, we decompose it by nonintersecting diagonals into triangles obtaining a tessellation with triangular faces whose vertices are the centers $O_{1}, O_{2}, \ldots$.

Let $O_{i}, O_{j}, O_{k}$ be a face of this tessellation, $\Delta_{i j k}$ its area and $\alpha_{i}, \alpha_{j}, \alpha_{k}$ the angles at $O_{i}, O_{j}, O_{k}$. We state that the density

$$
d=\frac{\alpha_{i} r_{i}^{2}+\alpha_{j} r_{j}^{2}+\alpha_{k} r_{k}^{2}}{2 \Delta_{i j k}}
$$

of the circles with respect to the triangle $O_{i} O_{j} O_{k}$ satisfies the inequality

$$
d \leqq 2 \pi / \sqrt{ } 3 .
$$

We consider three circles with variable centers $A, B, C$ and variable radii $r_{A}, r_{B}, r_{C}$ satisfying the same conditions as the circles $c_{i}, c_{j}, c_{k}$. Let us recapitulate these conditions.

1. The center-condition, involved in the definition of a Minkowskian circle-arrangement, can be expressed in terms of the sides $a, b, c$ of the triangle $A B C$. Supposing $a \leqq b \leqq c$, we have $r_{A} \leqq b, r_{B} \leqq a, r_{C} \leqq a$.

2. The intersection-condition, which follows from the construction of the above triangulation, says that the intersection of the three circles must contain a circle touching each of the three circles. This condition implies that the circle with center $C$ cannot completely contain the intersection of the circles with radii $a$ and $b$ centered at $B$ and $A$, respectively.

We shall show that under these conditions

$$
\frac{\alpha r_{A}^{2}+\beta r_{B}^{2}+\gamma r_{C}^{2}}{a b \sin \gamma} \leqq \frac{2 \pi}{\sqrt{ } 3},
$$

where $\alpha, \beta, \gamma$ are the angles of $A B C$. First we consider the

Case $\alpha+\beta \geqq \pi / 3$. Here we use the center-condition only, in view of which

$$
\begin{aligned}
\frac{\alpha r_{A}^{2}+\beta r_{B}^{2}+\gamma r_{C}^{2}}{a b \sin \gamma} & \leqq \frac{\alpha b^{2}+\beta a^{2}+\gamma a^{2}}{a b \sin \gamma}=\frac{\alpha}{\sin \gamma} \frac{b}{a}+\frac{\pi-\alpha}{\sin \gamma} \frac{a}{b} \\
& =\frac{(\pi-\alpha) \sin ^{2} \alpha+\alpha \sin ^{2} \beta}{\sin \alpha \sin \beta \sin (\alpha+\beta)}=f(\alpha, \beta) .
\end{aligned}
$$


We claim that for $0<\alpha \leqq \beta<\pi / 2$

$$
f(\alpha, \beta) \leqq f\left(\frac{\alpha+\beta}{2}, \frac{\alpha+\beta}{2}\right)=\frac{\pi}{\sin (\alpha+\beta)} .
$$

This inequality is equivalent with

$$
h(\beta)=(\pi-\alpha) \sin ^{2} \alpha+\alpha \sin ^{2} \beta-\pi \sin \alpha \sin \beta \leqq 0,
$$

which is true, since $h(\alpha)=0$ and

$$
h^{\prime}(\beta) / \cos \beta=2 \alpha \sin \beta-\pi \sin \alpha<2 \alpha-\pi \sin \alpha<0 .
$$

The stipulation $a \leqq b \leqq c$ implies $\alpha \leqq \beta \leqq \gamma=\pi-\alpha-\beta$. Thus $\pi / 3$ $\leqq \alpha+\beta \leqq 2 \pi / 3$, in consequence of which

$$
f(\alpha, \beta) \leqq \frac{\pi}{\sin (\alpha+\beta)} \leqq \frac{2 \pi}{\sqrt{ } 3} .
$$

Case $\alpha+\beta<\pi / 3$. We reflect the triangle $A B C$ in the line $A B$, obtaining the triangle $A B C^{\prime}$. As a consequence of the intersection-condition we have now $r_{C}<C C^{\prime}=\bar{c}$. Let $c_{A}, c_{B}, c_{C}, c_{C^{\prime}}$ be the circles with radii $b, a, \bar{c}, \bar{c}$ centered at $A, B, C, C^{\prime}$, respectively. We must estimate the density of these four circles with respect to the quadrangle $A C B C^{\prime}$ (which equals the density of $c_{A}, c_{B}, c_{C}$ with respect to $A B C$ ). But the results obtained in the case $\alpha+\beta \geqq \pi / 3$ show that both the density of $c_{A}, c_{C}, c_{C^{\prime}}$ with respect to $A C C^{\prime}$ and the density of $c_{B}, c_{C}$, $c_{C^{\prime}}$ with respect to $B C C^{\prime}$ are $\leqq 2 \pi / \sqrt{ } 3$. It follows that so is the density of the four circles with respect to the quadrangle.

This completes the proof of the inequality $d \leqq 2 \pi / \sqrt{ } 3$.

Let $O$ be a fixed point of the plane and $C(\mathrm{P})$ a circle of radius $\mathrm{P}$ centered at $O$. Again let $\sum_{\mathrm{P}}$ denote a summation extending either over those circles $C_{i}$ or over those triangles $O_{i} O_{j} O_{k}$ of the tessellation constructed above which are completely contained in $C(\mathrm{P})$. Since the circles $c_{i}, c_{j}, c_{k}$ have inner points in common, $O_{i} O_{j}<r_{i}+r_{j} \leqq r_{i}+R$ and $O_{i} O_{k}<r_{i}+r_{k} \leqq r_{i}+R$. Hence, if $c_{i}$ is contained in $C(\mathrm{P})$, then $O_{i} O_{j} O_{k}$ is contained in $C(\mathrm{P}+R)$. Thus

$$
\sum_{\mathrm{P}} \pi r_{i}^{2}<\frac{1}{2} \sum_{\mathrm{P}+R}\left(\alpha_{i} r_{i}^{2}+\alpha_{j} r_{j}^{2}+\alpha_{k} r_{k}^{2}\right) \leqq \frac{2 \pi}{\sqrt{ } 3} \sum_{\mathrm{P}+R} \Delta_{i j k}<\frac{2 \pi^{2}}{\sqrt{ } 3}(\mathrm{P}+R)^{2}
$$

showing that the (upper) density

$$
\bar{d}=\limsup _{\mathrm{P} \rightarrow \infty} \frac{1}{\pi \mathrm{P}^{2}} \sum_{\mathrm{P}} \pi r_{i}^{2}
$$

of the circles satisfies the inequality $\bar{d} \leqq 2 \pi / \sqrt{ } 3$. 
We finish with an outline of the proof of the following

THEOREM 2. If a finite set of circles form a Minkowskian arrangement then the density of the circles in their union cannot exceed $2 \pi / \sqrt{ } 3$.

We add to the circles infinitely many congruent circles of radius $\epsilon$ so as to obtain a Minkowskian arrangement of circles covering the plane completely. We construct the triangulation used in the proof of Theorem 1 and add the inequalities

$$
\frac{1}{2}\left(\alpha_{i} r_{i}^{2}+\alpha_{j} r_{j}^{2}+\alpha_{k} r_{k}^{2}\right) \leqq \frac{2 \pi}{\sqrt{ } 3} \Delta_{i j k}
$$

for all triangles at least one vertex of which coincides with the center of one of the original circles. Letting $\epsilon \rightarrow 0$, we obtain

$$
T \leqq \frac{2 \pi}{\sqrt{ } 3} U
$$

where $T$ is the total area of the circles and $U$ is the area of their union.

Although in this inequality the constant $2 \pi / \sqrt{ } 3$ cannot be replaced by a smaller one, equality can never hold. In a subsequent paper we intend to discuss a sharpening of the inequality $T / U<2 \pi / \sqrt{ } 3$ in which equality can be attained in various cases.

UNIVERSITY OF WISCONSIN 